\title{
Intracranial Peripheral-Type Primitive Neuroectodermal Tumor
}

\author{
-Case Report- \\ Yuichi FURUNO, Shinjitsu NISHIMURA, Hironaga KAMIYAMA, \\ Yoshihiro Numagami, Atsushi SAITO, \\ Mitsuomi KAIMORI*, and Michiharu NISHIJIMA
}

Departments of Neurosurgery and ${ }^{*}$ Pathology, Aomori Prefectural Central Hospital, Aomori

\begin{abstract}
A 15-year-old man presented with headache. Magnetic resonance (MR) imaging revealed a large extraaxial tumor with cyst at the right frontotemporal region. The solid part of the tumor was homogeneously enhanced on $T_{1}$-weighted MR imaging after injection of gadolinium. Digital subtraction angiography of the external carotid artery revealed sunburst appearance corresponding to the tumor, which was fed by the right middle meningeal artery. His headache worsened and computed tomography revealed enlargement of the tumor and intracystic hemorrhage, so emergent operation was performed. At surgery, the tumor strongly adhered to the dural membrane, and was obviously extraaxial. The tumor and cyst were gross totally removed. The attachment site at the dura mater was resected. Histological examination showed solid growth of small round cells with uniform round nuclei and minimal cytoplasm. Immunohistochemical staining showed the cells were positive for MIC-2 (CD99). The MIB-1 labeling index was $53 \%$. The histological diagnosis was peripheral-type primitive neuroectodermal tumor (pPNET). Following surgery, radiation therapy and chemotherapy were given. Ewing's sarcoma and pPNET form a family of small round cell tumors arising in the bone or soft tissue. MIC-2 is a useful marker in the differential diagnosis. Good prognosis may be attained if complete surgical excision of intracranial pPNET is achieved.
\end{abstract}

Key words: peripheral-type primitive neuroectodermal tumor, MIC-2 antigen, cystic meningioma

\section{Introduction}

Primitive neuroectodermal tumor (PNET) was first described by Hart and Earle ${ }^{6)}$ in 1973 as predominantly undifferentiated tumor of the cerebrum which did not meet the diagnostic criteria for neuroblastoma, ependymoblastoma, medulloblastoma, or pineal parenchymal tumors. PNET is a malignant, embryonal neoplasm consisting of poorly differentiated neuroepithelial cells occurring in children and young adults. ${ }^{5}$ The first case was found in association with the ulnar nerve ${ }^{19)}$ but cases are known in almost every organ of the body. Recently, these tumors were divided into central nervous system PNET (cPNET), originating in the central or sympathetic nervous system, and peripheral-type PNET (pPNET), originating in the soft tissue or bone. These two types have different prognoses. ${ }^{2)}$ pPNET and Ewing's sarcoma form a family of tumors with a common cytogenetic translocation $t(11 ; 22)(q 24 ; q 12)$ and the same highly consistent pattern of protooncogene expression. MIC-2 is a specific marker for this family and is useful in the differential diagnosis of the two types of PNETs. pPNET could theoretically arise within the intracranial cavity. However, intracranial pPNET is rare, with only 15 cases of pPNET originating in the intracranial soft tissue. . $^{1,37-11,14-18,20)}$

We describe a case of intracranial pPNET mimicking a cystic meningioma.

\section{Case Report}

A 15-year-old man presented to another clinic with a

Received June 6, 2007; Accepted November 6, 2007

Author's present address: Y. Furuno, M.D., Department of Neurosurgery, Kyoto Prefectural University of Medicine, Kyoto, Japan. 

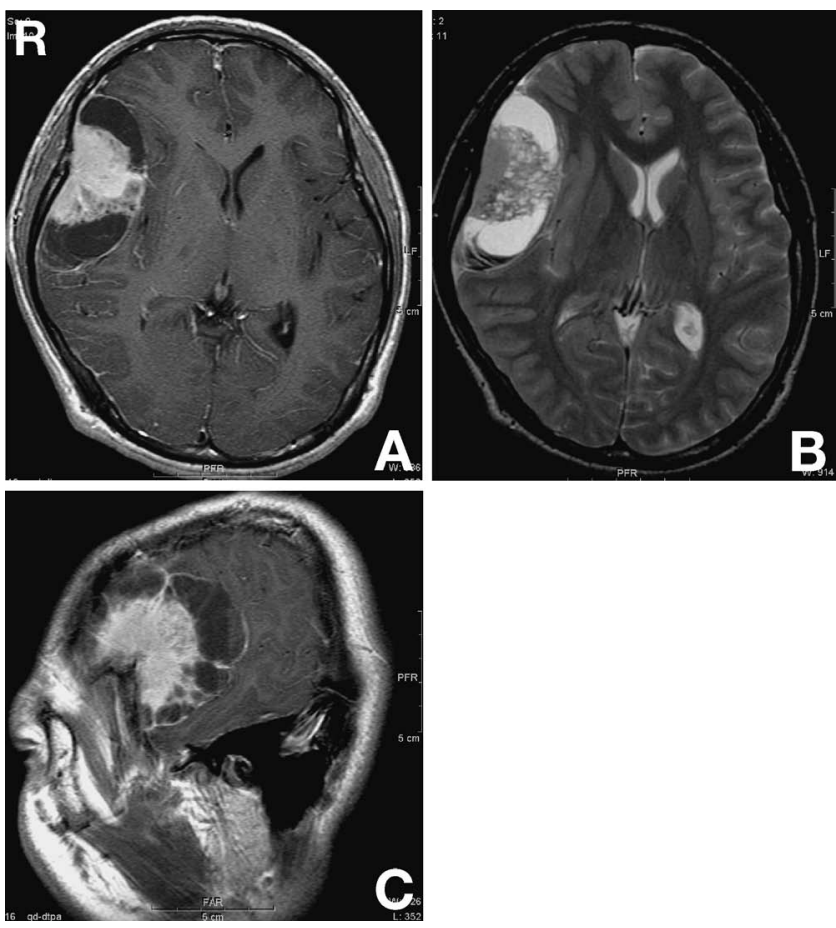

Fig. 1 Preoperative axial $\mathrm{T}_{1}$-weighted magnetic resonance image with gadoliniumdiethylenetriaminepenta-acetic acid (GdDTPA) (A), axial $T_{2}$-weighted image (B), and sagittal $T_{1}$-weighted image with Gd-DTPA (C) demonstrating a well-enhanced extraaxial tumor with cyst.

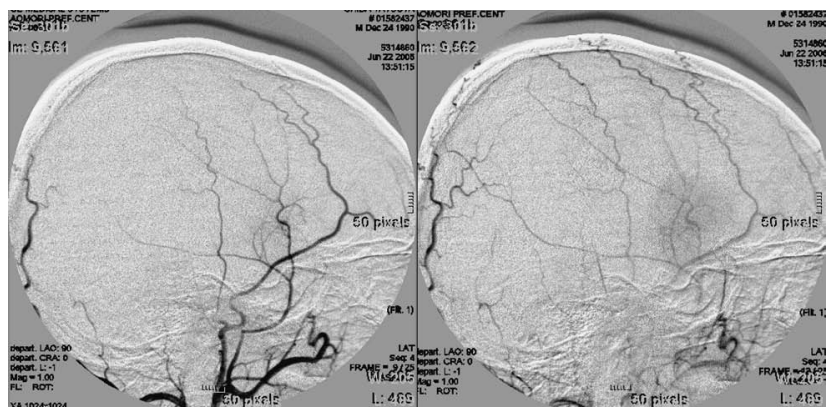

Fig. 2 Digital subtraction angiograms demonstrating the tumor was fed by the right middle meningeal artery with sunburst appearance.

1-week history of headache. Magnetic resonance (MR) imaging revealed a large intracranial mass lesion, and he was referred to our hospital. On admission, the patient was alert and oriented. Neurological examination revealed no deficits. Laboratory data were within normal limits. MR imaging revealed a $6.6 \times 2.6 \times 6.0-\mathrm{cm}$ extraaxial tumor with cyst in the right frontotemporal region (Fig. 1). The

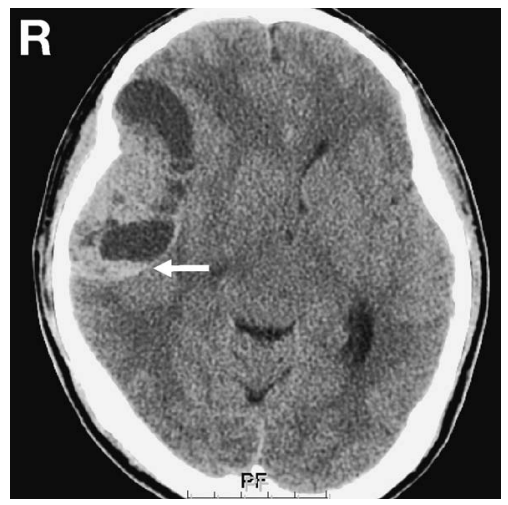

Fig. 3 Axial computed tomography scan demonstrating enlargement of the tumor and the cyst, and intracystic hemorrhage (arrow).

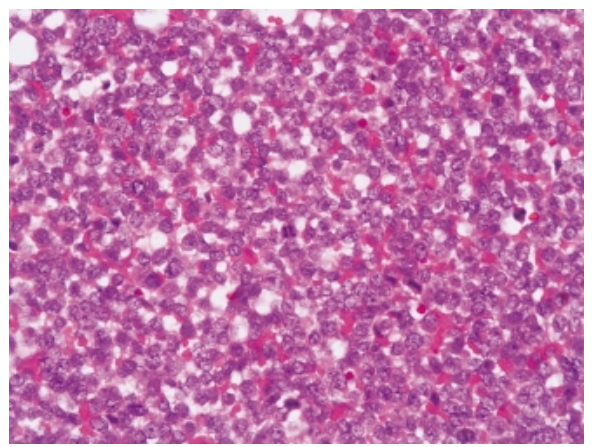

Fig. 4 Photomicrograph of tissue section demonstrating small round cells and mitoses. Hematoxylin and eosin stain, original magnification $\times \mathbf{2 0 0}$.

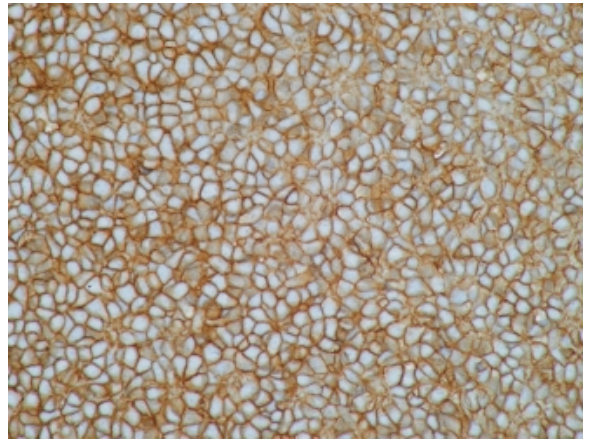

Fig. 5 Photomicrograph revealing diffuse immunohistochemical staining of the tumor cells for antibodies against MIC-2 protein. Original magnification $\times \mathbf{2 0 0}$.

solid part of the tumor was homogeneously enhanced on $\mathrm{T}_{1}$-weighted $\mathrm{MR}$ imaging after injection of gadolinium. No perifocal edema was noted. Digi- 
tal subtraction angiography of the external carotid artery revealed sunburst appearance corresponding to the tumor, and identified the feeding artery as the right middle meningeal artery (Fig. 2).

Preoperative embolization and surgery were planned under a diagnosis of cystic meningioma. However, his headache worsened on the 12th day of hospitalization. Computed tomography (CT) revealed enlargement of the tumor and intracystic hemorrhage (Fig. 3), so emergent operation was performed on the same day. The patient underwent right frontotemporal osteoplastic craniotomy, followed by removal of the tumor. The tumor strongly adhered to the dural membrane, and was obviously extraaxial. The cyst was readily removed leaving the arachnoid membrane intact. The tumor and cyst were gross totally removed. The attachment site at the dura mater was resected.

Histological examination showed solid growth of small round cells with uniform round nuclei and minimal cytoplasm (Fig. 4). Immunohistochemical staining showed the cells were positive for MIC-2 (CD99) (Fig. 5), carcinoembryonic antigen, synaptophysin, and S-100 protein, but negative for leukocyte common antigen and glial fibrillary acidic protein. The MIB-1 labeling index was 53\%. The final diagnosis was pPNET. MR imaging of the spine was normal, and examination of the cerebrospinal fluid was negative for malignant cells. No tumor was visualized by CT of the chest and abdomen. Following surgery, we started radiation therapy and chemotherapy. He is alive with no evidence of disease at 6 months following diagnosis.

\section{Discussion}

Table 1 shows the 16 reported cases of intracranial pPNET including the present case. The eight male and eight female patients were aged between 5 months and 67 years (mean 19 years). The tumors were located in the supratentorial dura mater in seven cases including our case, frontal skull base in two cases, cavernous sinus in one case, cerebellopontine angle or tentorium in five cases, and frontal lobe in one case. Gross total removal was performed in 10 cases, partial removal in three cases, and open biopsy in two cases. The follow-up period was 9 days to 10 years (mean 2.6 years), and three patients died.

pPNET and Ewing's sarcoma were previously believed to be different tumors. However, a specific balanced translocation involving the Ewing's sarcoma gene on chromosome 22 has been demonstrated in both of these tumors and pPNET is now considered part of the spectrum of round cell sarcoma, in- cluding Ewing's sarcoma. ${ }^{4)}$ The most common translocation is $\mathrm{t}(11 ; 22)(\mathrm{q} 24 ; \mathrm{q} 12) .{ }^{10,13)}$ Immunoreactivity to HBA-71, which recognizes the cell surface glycoprotein p30/32 (a product of the MIC2 gene), is a highly sensitive histochemical marker of this family of tumors. ${ }^{3,10,13)}$ The diagnosis of cPNET can be confirmed by absence of MIC2 expression and $\mathrm{t}(11 ; 22)$ translocation. MIC-2 is a specific marker for pPNET/Ewing's sarcoma family and is useful for the differential diagnosis of these two types of tumor. To make a diagnosis more exactly, it is recommended to confirm the presence of $t(11 ; 22)(q 24 ; q 12)$ translocation by reverse transcriptase-polymerase chain reaction and fluorescent in situ hybridization, if it is possible.

The neuroimaging differential diagnosis of pPNET includes meningioma, neurinoma, metastasis, and lymphoma, but no characteristic signs are known. pPNET was misdiagnosed as meningioma and hemangiopericytoma, so chemotherapy and radiation therapy were delayed and only performed for local recurrence and metastasis 9 years after the first diagnosis. ${ }^{17)}$ pPNET should be considered in the differential diagnosis of meningeal tumor, because surgical treatment may require early additional chemotherapy, in contrast to meningioma.

In our case, tumor was associated with intracystic hemorrhage. In Cases 10 and 13, the tumors also had hemorrhagic changes on MR imaging. Although the frequency of tumoral hemorrhage in pPNET is unknown, we should keep in mind that tumoral hemorrhage may cause symptomatic deterioration in patients with pPNET.

pPNET requires multimodality treatment. Surgery is important for tumor control. Wide surgical resection margins at the first surgery have markedly reduced local recurrences. The prognosis of pPNET has markedly improved with adjuvant chemotherapy and radiotherapy. ${ }^{7,12)}$ Long-term survival is uncommon among patients with cPNET, and many die within 1 year of diagnosis despite combined surgery, radiation therapy, and chemotherapy. ${ }^{3,6,10)}$ The median survival of cPNET was reported as 23 months, and 2,3 , and 5 -year survivals as $50 \%, 34 \%$, and $18 \%$, respectively. ${ }^{5)}$ In our review of 16 cases, 11 patients were followed up over 1 year and only one patient died within 1 year (1-year survival of 91\%). Eight patients were followed up over 2 years, and two died within 2 years (2-year survival of $75 \%$ ). Four patients were followed up over 5 years, and two died within 5 years (5-year survival of 50\%) (Table 1). The clinical course of intracranial pPNET is clearly more favorable than that of cPNET, so distinction of these two types is very important.

This case was first diagnosed as cystic meningio- 


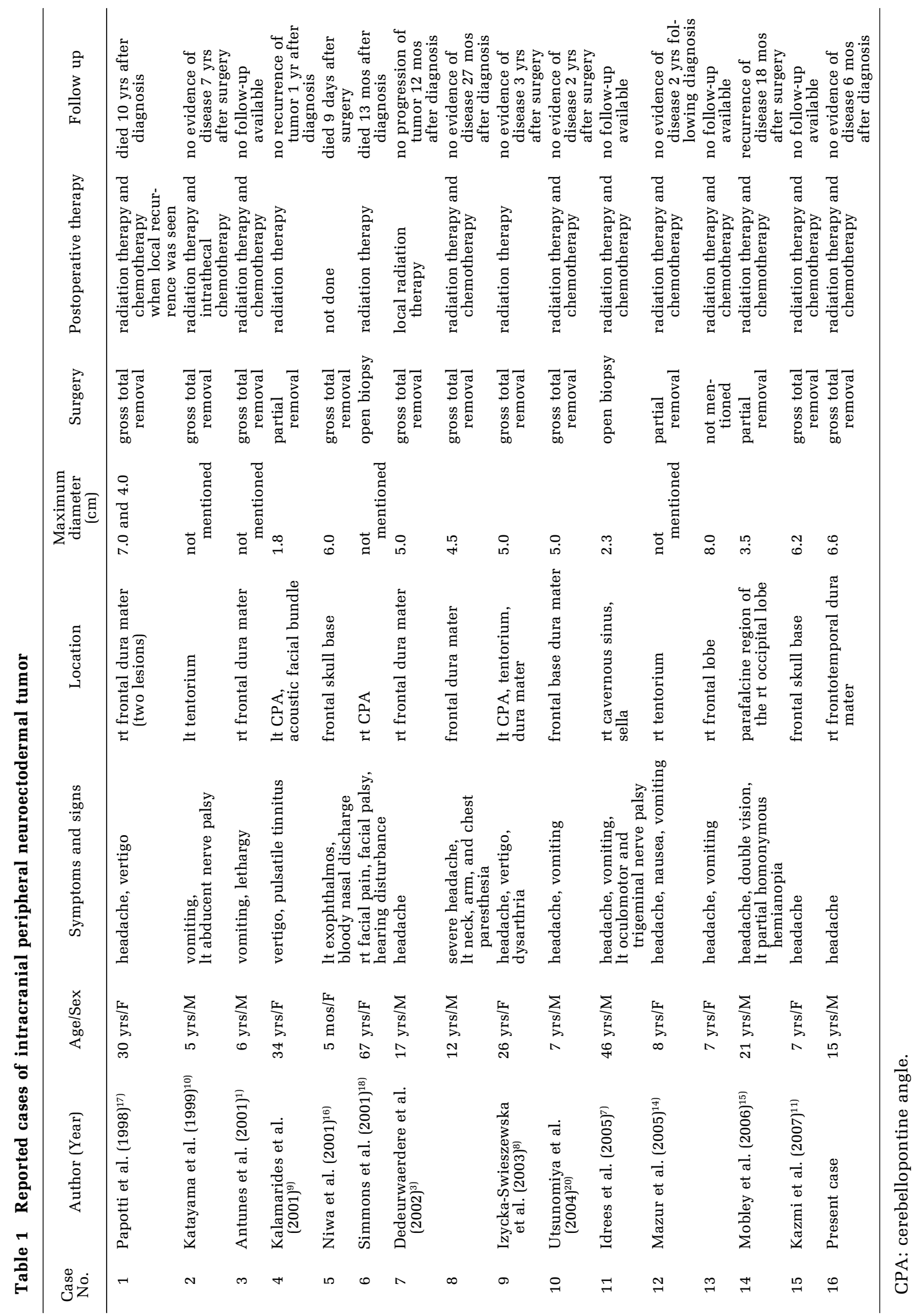


ma. At surgery, the tumor was gross totally resected and the attachment site at the dura mater was resected with a wide surgical margin. The correct diagnosis of pPNET was then established, and radiation therapy and chemotherapy were given. Good prognosis is expected, but recurrence or metastases of the tumor remain possible.

\section{References}

1) Antunes NL, Lellouch-Tubiana A, Kalifa C, Delattre O, Pierre-Kahn A, Rosenblum MK: Intracranial Ewing sarcoma/'peripheral' primitive neuroectodermal tumor of dural origin with molecular genetic confirmation. J Neurooncol 51: 51-56, 2001

2) Batsakis JG, El-Naggar AK: Ewing's sarcoma and primitive neuroectodermal tumors: cytogenetic cynosures seeking a common histogenesis. Adv Anat Pathol 4: 207-220, 1997

3) Dedeurwaerdere F, Giannini C, Sciot R, Rubin BP, Perilongo G, Borghi L, Ballotta ML, Cornips E, Demunter A, Maes B, Dei Tos AP: Primary peripheral PNET/Ewing's sarcoma of the dura: a clinicopathologic entity distinct from central PNET. Mod Pathol 15: 673-678, 2002

4) Dehner LP: Peripheral and central primitive neuroectodermal tumors. A nosologic concept seeking a consensus. Arch Pathol Lab Med 110: 997-1005, 1986

5) Dirks PB, Harris L, Hoffman HJ, Humphreys PR, Drake JM, Rutka JT: Supratentorial primitive neuroectodermal tumors in children. J Neurooncol 29: 75-84, 1996

6) Hart $\mathrm{MN}$, Earle KM: Primitive neuroectodermal tumors of the brain in children. Cancer 32: 890-897, 1973

7) Idrees M, Gandhi C, Betchen S, Strauchen J, King W, Wolfe D: Intracranial peripheral primitive neuroectodermal tumors of the cavernous sinus: a diagnostic peculiarity. Arch Pathol Lab Med 129: e11-15, 2005

8) Izycka-Swieszewska E, Debiec-Rychter M, Kloc W: Primitive neuroectodermal tumor in the cerebellopontine angle with isochromosome 17q presenting as meningioma in a woman 26 years of age. Clin Neuropathol 22: 66-70, 2003

9) Kalamarides M, Dewolf E, Couvelard A, Shahidi A, Bouccara D, Cyna-Gorse F, Rey A, Sterkers O: Extraaxial primitive neuroectodermal tumor mimicking a vestibular schwannoma: diagnostic and therapeutic difficulties. Report of two cases. J Neurosurg 94: 612-616, 2001

10) Katayama $Y$, Kimura S, Watanabe $T$, Yoshino A, Koshinaga M: Peripheral-type primitive neuroec- todermal tumor arising in the tentorium. Case report. J Neurosurg 90: 141-144, 1999

11) Kazmi SA, Perry A, Pressey JG, Wellons JC, Hammers Y, Palmer CA: Primary Ewing sarcoma of the brain: a case report and literature review. Diagn Mol Pathol 16: 108-111, 2007

12) Kushner BH, Hajdu SI, Gulati SC, Erlandson RA, Exelby PR, Lieberman PH: Extracranial primitive neuroectodermal tumors. The Memorial Sloan-Kettering Cancer Center experience. Cancer 67: 18251829, 1991

13) Llombart-Bosch A, Navarro S: Immunohistochemical detection of EWS and FLI-1 proteins in Ewing sarcoma and primitive neuroectodermal tumors: comparative analysis with CD99 (MIC-2) expression. Appl Immunohistochem Mol Morphol 9: 255-260, 2001

14) Mazur MA, Gururangan S, Bridge JA, Cummings TJ, Mukundan S, Fuchs H, Larrier N, Halperin EC: Intracranial Ewing sarcoma. Pediatr Blood Cancer 45: 850-856, 2005

15) Mobley BC, Roulston D, Shah GV, Bijwaard KE, McKeever PE: Peripheral primitive neuroectodermal tumor/Ewing's sarcoma of the craniospinal vault: case reports and review. Hum Pathol 37: 845-853, 2006

16) Niwa J, Shimoyama N, Takahashi Y: Primitive neuroectodermal tumor involving the frontal skull base in an infant. Childs Nerv Syst 17: 570-574, 2001

17) Papotti M, Abbona G, Pagani A, Monga G, Bussolati G: Primitive neuroectodermal tumor of the meninges: A histological, immunohistochemical, ultrastructural, and cytogenetic study. Endocr Pathol 9: 275-280, 1998

18) Simmons MA, Luff DA, Banerjee SS, Ramsden RT: Peripheral primitive neuroectodermal tumour (pPNET) of the cerebellopontine angle presenting in adult life. J Laryngol Otol 115: 848-852, 2001

19) Stout AP: A tumor of the ulnar nerve. Proc $N Y$ Pathol Soc 12: 2-12, 1918

20) Utsunomiya A, Uenohara H, Suzuki S, Shimosaka $S$, Numagami Y, Nishimura S, Nishino A, Suzuki H, Sakurai Y: [A case of peripheral-type primitive neuroectodermal tumor arising in the dura mater at the frontal base]. No To Shinkei 56: 237-241, 2004 (Jpn)

Address reprint requests to: Yuichi Furuno, M.D., Department of Neurosurgery, Kyoto Prefectural University of Medicine, 465 Kajii-cho, Kawaramachi-Hirokoji, Kamigyo-ku, Kyoto 602-8566, Japan.

e-mail: yfuruno@koto.kpu-m.ac.jp 